\title{
A CASE IN WHICH IRREDUCIBILITY OF AN ANALYTIC GERM IMPLIES IRREDUCIBILITY OF THE TANGENT CONE ${ }^{1}$
}

\author{
RICHARD DRAPER
}

\begin{abstract}
There are simple examples in which a variety is irreducible at a point but has a reducible tangent cone. The following theorem is proved. If $X_{p}$ is an irreducible analytic germ and if the Jacobian ideal beomes principal on the normalization then the tangent cone of $X$ at $p$ is irreducible. If, moreover, the singular set of $X$ is a manifold at $p$ then $X$ is Whitney $a, b$-regular along the singular set at $p$.
\end{abstract}

Let $X$ be a pure $r$-dimensional analytic subset of open $U$ in $C^{n}$. $\mathcal{O}^{n}$ denotes the holomorphic structure sheaf on $U, I$ denotes the (self-radical) ideal sheaf defining $X$ in $U$, and $\mathcal{O}$ denotes the resulting (reduced) holomorphic structure on $X$. Let $T=\left(T_{1}, \cdots, T_{n}\right)$ be coordinates on $C^{n}$. Hereafter $J$ denotes the Jacobian ideal of $X$ on $X$. It is a coherent sheaf of ideals whose stalk at $p$ is obtained as follows. Take the $\infty \times n$ matrix whose entries are of the form $\left(\partial f /\left.\partial T_{j}\right|_{X}, j=1, \cdots, n, f \in I_{p}\right) . J_{p}$ is the ideal in $\mathcal{O}_{p}$ generated by the $(n-r) \times(n-r)$ subdeterminants of this matrix. It is sufficient to restrict $f$ to a system of generators for $I_{p}$ in which case one obtains a system of generators for $J_{p}$. Thus we can assume that $f$ belongs to a finite set $\mathscr{F}$ of generators for $I_{p}$. By replacing $U$ by a smaller open set we may also assume $f$ is holomorphic on $U, \forall f \in \mathscr{F},\left\{f_{a}, f \in \mathscr{F}\right\}$ generates $I_{q}$, and the $(n-r) \times(n-r)$ subdeterminants obtained from the matrix

$$
\left(\partial f /\left.\partial T_{j}\right|_{X}, j=1, \cdots, n, f \in \mathscr{F}\right)
$$

have germs at $q$ which generate the ideal $J_{q}$ for all $q$ in $X$. Let $t=\#(\mathscr{F})$.

The singular set of $X$, hereafter denoted $\operatorname{Sg}(X)$, is the locus of $J$.

Let $\pi:\left(X^{\prime}, \mathcal{O}^{\prime}\right) \rightarrow(X, \mathcal{O})$ denote the normalization of $X$. The following are

Received by the editors July 17, 1972.

AMS (MOS) subject classifications (1970). Primary 14B05, 32B15.

Key words and phrases. Tangent cone, Jacobian ideal, normalization, Whitney $a, b$. regularity.

${ }^{1}$ This work was done while the author was a visiting staff member at Purdue University. The manuscript was prepared while the author was supported by NSF Grant GP-20139 Amendment Number 2 at the University of Notre Dame. The manuscript was revised while the author was a staff member at George Mason University.

(c) American Mathematical Society 1973 
in natural one-to-one correspondence: $\left\{p_{1}^{\prime}, \cdots, p_{k}^{\prime}\right\}=\pi^{-1}(p),\left\{\mathfrak{p}_{1}, \cdots, \mathfrak{p}_{k}\right\}=$ minimal primes of $\mathcal{O}_{p},\left\{X_{1}, \cdots, X_{k}\right\}=$ irreducible components of the germ of $X$ at $p$. We say $J \mathcal{O}^{\prime}$ is locally principal over $p$ if and only if $J_{p} \mathcal{O}_{p_{\lambda^{\prime}}}^{\prime}$ is principal for $\lambda=1, \cdots, k$. This is an open condition on $X$.

Let $T(X)=\left\{(p, u) \in X \times C^{n} \mid \sum f_{j}(p) u_{j}=0 \forall f \in \mathscr{F}\right\}$ where $f_{j}=\partial f / \partial T_{j}$. $T(X, p)$ denotes the fiber of the projection of $T(X)$ to $X$. The singular points of $X$ are those for which $\operatorname{dim} T(X, p)>r$. We let $Y$ denote the set of singular points. $C_{4}(X)$ is the closure in $T(X)$ of $T(X-Y) . C_{4}(X, p)$ is the fiber of $C_{4}(X)$ over $p$. It is the union of a collection of $r$-dimensional subspaces of $C^{n}$. Let $G^{n-1, r-1}$ be the Grassmann variety of $r$-dimensional subspaces of $C^{n}$. If $q$ is simple on $X, T(X, q)$ has a unique point $\tau(X, q)$ in $G^{n-1, r-1}$. Let $\tau(X)$ denote the closure of $\{(q, \tau(X, q)) \mid q \in X-Y\}$ in $X \times G^{n-1, r-1}$. Let $\tau(X, p)$ denote the fiber of the projection to $X$. See [6, Theorem 5.1, p. 218 and Theorem 7.1, p. 224]. There is a natural map $\varphi: C_{4}(X) \rightarrow \tau(X)$ which commutes with the projections to $X$ and makes $C_{4}(X)$ a fiber space of constant fiber dimension $n-r$ over $\tau(X) . \tau(X) \rightarrow X$ is a proper modification of $X$ which is an isomorphism exactly over $X-Y$. Denote the structure sheaf on $\tau(X)$ by $\widetilde{\mathcal{O}}$.

Let $\left(B_{J}(X), \hat{\mathcal{O}}\right)$ denote the blowing-up of $X$ along $J$. This is a subspace of $X \times P^{N}$ which can be realized as follows. Let $\mathscr{I}$ denote the functions on $X$ obtained from $(*)$. These are determinants which we enumerate in the following way for our subsequent convenience. Let $\mathscr{F}=\left(f_{1}, \cdots, f_{t}\right)$ and let $\lambda=\left(\lambda_{1}, \cdots, \lambda_{n-r}\right)$. By $J_{\lambda}$ we mean the tuple obtained from $(*)$ by taking subdeterminants from the $(n-r) \times n$ submatrix of $\left({ }^{*}\right)$ whose rows involve the functions $f_{\lambda_{j}}, j=1, \cdots, n-r$. Let $J_{\lambda}^{k}, k=1, \cdots,\left(\begin{array}{c}n \\ n-r\end{array}\right)$ denote the entry of this tuple ordered in some fixed manner. Let $\mathscr{I}=\left(\cdots, J_{\lambda}, \cdots\right)$ be a fixed ordering of the tuples $J_{\lambda}$. Introduce indeterminants $Z_{\lambda}^{k}$ ordered in the same manner. Then $\left(B_{J}(X), \hat{\mathcal{O}}\right)$ is the analytic space defined by the equations $\left\{Z_{\lambda}^{k} J_{\mu}^{j}-Z_{\mu}^{j} J_{\lambda}^{k}=0\right.$, all $\lambda, \mu, k, j$ subject to the above conventions $\}$ in $X \times$ $P^{N-1}$ where the $Z_{\lambda}^{k}$ are homogeneous coordinates on $P^{N-1}$ and $N=$ $\left(\begin{array}{c}t \\ n-1\end{array}\right)\left(\begin{array}{c}n \\ n-r\end{array}\right) . B_{J}(X) \rightarrow X$ blows up exactly over $X-Y$. We denote the fiber of this projection by $B_{J}(X, p)$.

The tangent cone to $X$ at $p$, denoted $C(X, p)$, is $\left\{u \in C^{n} \mid f^{*}(u)=\right.$ $\left.0 \forall f \in I_{p}\right\}$ where $f^{*}$ denotes the leading form at $p$ of $f$. Since $G\left(\mathcal{O}_{p}\right)$, the associated graded ring of $\mathcal{O}_{p}$ with respect to its maximal ideal, is isomorphic to $C[T] / A$ where $A$ is the ideal generated by $\left\{f^{*}(T) \mid f \in I_{p}\right\}$, there is a oneto-one correspondence between the components of the cone $C(X, p)$ and the minimal primes of $G\left(\mathcal{O}_{p}\right)$. We use this and the fact, $[6,(3.1)$, p. 212], that $C_{4}(X, p) \supset C(X, p)$ to prove the theorem of this paper.

Proposition 1. With the notation and assumptions of the preceding paragraphs, the following are equivalent: 
(i) $\operatorname{dim} C_{4}(X, p)=r$,

(ii) $\tau(X, p)$ is finite,

(iii) $B_{J}(X, p)$ is finite,

(iv) $J$ is locally principal over $p$ on $\left(X^{\prime}, \mathcal{O}^{\prime}\right)$,

(v) $C_{4}(X, p)=C(X, p)$.

Moreover, if these conditions hold then $\# \tau(X, p) \leqq \# B_{J}(X, p) \leqq \#\{$ irreducible components of $\left.X_{p}\right\}$ and $C(X, p)$ is a union of $\# \tau(X, p) r$-dimensional subspaces of $C^{n}$.

Proof. As remarked earlier $C_{4}(X, p)$ is the union of $r$-dimensional subspaces of $C^{n} \cdot \tau(X, p)$ is the union of the Grassmann coordinates of these subspaces. Both $C_{4}(X, p)$ and $\tau(X, p)$ are algebraic sets. Clearly $\operatorname{dim} C_{4}(X, p)=r$ if and only if $\tau(X, p)$ is finite which proves the equivalence of (i) and (ii).

Indices have the meanings established in the description of $B_{J}(X)$. We will show that to each point of $\tau(X, p)$ there corresponds at least one and at most finitely many points of $B_{J}(X, p)$. (This is always true. Moreover, when $X$ is a complete intersection $(\tau(X), \widetilde{\mathcal{O}})$ and $\left(B_{J}(X), \hat{\mathcal{O}}\right.$ red $)$ are isomorphic. In [4, Remark (1.2), p. 3] it seems to be stated that they are always isomorphic. This does not appear to be true to us although we have been unable to produce a counterexample.) Let $(p, \alpha) \in \tau(X)$. Let $a$ be affine coordinates for $\alpha$. There exist simple points $p_{v} \rightarrow p, c_{v} \in C$, such that $c_{v} J_{\mu}\left(p_{v}\right) \rightarrow a$ for at least one $\mu$. Moreover, given any $\mu$ such that $J_{\mu}\left(p_{v}\right) \neq 0$ for almost all $v$ a sequence $d_{v}$ exists such that $d_{v} J_{\mu}\left(p_{v}\right) \rightarrow a$ on the subsequence of $\left\{p_{v}\right\}$ at which $J_{\mu}\left(p_{v}\right) \neq 0$. The reason for this is that, if $J_{\mu}\left(p_{v}\right) \neq 0$, then $J_{\mu}$ is (on the ray corresponding to) the Grassmann coordinates of $T\left(X, p_{v}\right)$. Consequently $c_{v}\left(\cdots, J_{\lambda}\left(p_{v}\right), \cdots\right)$ converges (on a subsequence of $\left.\left\{p_{v}\right\}\right)$ to a point $b=\left(\cdots, b_{\lambda}, \cdots\right)$ with the following properties (where $\beta$ denotes the point of $P^{N-1}$ corresponding to $b$ ): $(p, \beta) \in$ $B_{J}(X)$; if $b_{\lambda} \neq 0$ then $b_{\lambda}$ is proportional to $a ; b_{\lambda} \neq 0$ for some $\lambda$. Thus, given $(p, \alpha)$ in $\tau(X)$ we have constructed a point $(p, \beta)$ in $B_{J}(X)$. How many such points can exist? The construction depends not only on $(p, \alpha)$ but also on the sequence $\left\{p_{v}\right\}$. It is conceivable that for a different sequence $\left\{q_{v}\right\}$, $\left\{\lambda \mid b_{\lambda} \neq 0\right\}$ could change yielding another point of $B_{J}(X, p)$ corresponding to $(p, \alpha)$. Since the only possible variation is in $\left\{\lambda \mid b_{\lambda} \neq 0\right\}$ and since there are only finitely many choices for the tuple $\lambda$, we conclude there are at most finitely many points on $B_{J}(X, p)$ for each point on $\tau(X, p)$. Consequently (ii) and (iii) are equivalent and the first inequality is proved.

Suppose $B_{J}(X, p)$ is finite. Since $B_{J}(X) \rightarrow X$ is a proper modification, $B_{J}(X, q)$ is finite for all $q$ near $p$ and there is a natural map $\theta:\left(X^{\prime}, \mathcal{O}^{\prime}\right) \rightarrow$ $\left(B_{J}(X), \hat{\mathcal{O}}\right)$ commuting with the projections to $X$ over a neighborhood of $p$. $\theta$ is proper and surjective. (This is the universal mapping property of 
normalization $\left[1,46.20\right.$, p. 456].) $J \hat{\mathcal{O}}$ is locally principal and $J \mathcal{O}^{\prime}$ is the pullback of $J \hat{\mathcal{O}}$ induced by $\theta$ so $J \mathcal{O}^{\prime}$ is locally principal which proves (iii) implies (iv) and the second inequality.

We also use $\theta$ to prove (iii) implies (v). Both $C_{4}(, p)$ and $C(, p)$ distribute across unions so we may assume $X_{p}$ is irreducible and only one point $p^{\prime}$ of $X^{\prime}$ lies over $p$. Hence, existence of $\theta$ shows that only one point is in $B_{J}(X, p)$. Consequently $\tau(X, p)$ consists of one point. Hence, $C_{4}(X, p)$ consists of a single plane of dimension $r$. Since every component of $C(X, p)$ has dimension $r$ (proving (v) implies (iii)) and $C_{4}(X, p) \supset C(X, p)$, it follows that they are equal. This proves that (iii) implies (v).

It remains to prove that (iv) implies (iii). If $J \mathcal{O}^{\prime}$ is locally principal over $p$ then there is a natural map $\psi:\left(X^{\prime}, \mathcal{O}^{\prime}\right) \rightarrow\left(B_{j}(X), \hat{\mathcal{O}}\right)$, which commutes with projections to $X$ over a neighborhood of $p . \psi$ is proper and surjective. (This is the universal mapping property of blowing-up [3, p. 123].) Since $X^{\prime}$ has finite fiber over $p, B_{J}(X, p)$ is finite. Q.E.D.

Corollary. Suppose $X_{j}$ denote the irreducible components of $X$ at $p$, $I_{p}(X), I_{p}\left(X_{j}\right)$, is the ideal defining $X$, resp. $X_{j}$, at $p$ and $J, J_{j}$, is the Jacobian ideal defined by $I_{p}(X)$, resp. $I_{p}\left(X_{j}\right)$. Then $J$ becomes locally principal over $p$ on the normalization of $X$ if and only if $J_{j}$ becomes principal over $p$ on the normalization of $X_{j}$ for all $j$.

Proof. $\quad C_{4}(X, p)=\bigcup C_{4}\left(X_{j}, p\right)$. Q.E.D.

THEOREM 2. Let $R$ be the local ring of a point $p$ on a reduced, pure $r$ dimensionalanalytic space $X$. Suppose that the Jacobian ideal becomes locally principal over $p$ on the normalization of $X$. Then each minimal prime ideal $P$ of $G(R)$ determines at least one minimal prime ideal $\mathfrak{p}$ of $R$ such that $P$ is the radical of $\operatorname{Ker}(G(R) \rightarrow G(R / \mathfrak{p}))$. Moreover, $G(R) / P$ is a polynomial ring in $r$ variables over $C$.

Proof. The hypothesis implies that $C(X, p)=C_{4}(X, p)$ and is a finite union of $r$-dimensional subspaces of $C^{n}$. These planes are in one-to-one correspondence with the minimal primes of $G(R)$. Let $L$ be the plane corresponding to $P$. Because $C(, p)$ distributes across unions, there is at least one irreducible component $Z$ of $X_{p}$ such that $L \subset C(Z, p)$. Applying the inequality of Proposition 1 we conclude that $\# \tau(Z, p)=1$ and $L=C(Z, p)$. $Z$ is determined by a minimal prime ideal of $R$ and $P$ is the radical of the kernel of $G(R) \rightarrow G(R / \mathfrak{p}) . G(R / \mathfrak{p})$ has as reduction a ring of polynomials in $r$ indeterminants because $L$ is a plane of dimension $r$. Since $G(R) \rightarrow$ $G(R / \mathfrak{p})$ is surjective $G(R) / P$ is isomorphic to the reduction of $G(R / \mathfrak{p})$. Q.E.D. 
COROllary. If, in addition to the hypothesis of the theorem, $R$ is a domain, then $G(R)$ has as reduction an integral domain.

Proof. (0) is the only minimal prime of $R$ so $G(R)$ can have only one minimal prime. Q.E.D.

EXAMPLES. The locus of $Y^{2}-X^{3}=0$ is an example showing that $G(R)$ need not be a domain.

In general the correspondence between primes of $G(R)$ and primes of $R$ is one-to-many, e.g. the locus of $Y\left(Y-X^{2}\right)=0$.

The locus of $X Y-Z^{3}=0$ shows that assuming $C(X, p)$ a union of planes and $X_{p}$ irreducible does not insure that $C(X, p)$ is irreducible.

Question. What condition together with irreducibility of $X_{p}$ insures irreducibility of $C(X, p)$ ? The condition given here seems overly strong since it insures that $C(X, p)$ is a plane, not just irreducible.

Recall the Whitney conditions $[6, \S 8]$. If $X$ is an analytic space, $Y$ is a manifold, and $p \in X \cap Y, X$ is said to be $a$-regular along $Y$ at $p$ if: whenever $\left\{p_{v}\right\} \in X-S g(X)$ with $p_{v} \rightarrow p$ and $T\left(X, p_{v}\right) \rightarrow T$ then $T \supset T(Y, p) . X$ is said to be $b$-regular along $Y$ at $p$ if: whenever $\left\{p_{v}\right\} \in X-\operatorname{Sg}(X),\left\{q_{v}\right\} \in Y,\left\{c_{v}\right\} \in C$ with $p_{v} \rightarrow p, q_{v} \rightarrow p, T\left(X, p_{v}\right) \rightarrow T$ and $c_{v}\left(p_{v}-q_{v}\right) \rightarrow v$ then $v \in T . X$ is said to be $a, b$-regular along $Y$ at $p$ if $X$ is both $a$-regular and $b$-regular along $Y$ at $p$. We can see that $J O^{\prime}$ locally principal over $p$ allows us to determine $\lim T\left(X, p_{v}\right)=T$. For the limit to exist, infinitely many $p_{v}$ must be on one component $X_{j}$ of $X$ at $p$ and $T=C\left(X_{j}, p\right)$. Thus $a$-regularity reduces to the simple property: every component of $C(X, p)$ contains $T(Y, p)$ at $p$ if $J \mathcal{O}^{\prime}$ is locally principal over $p$. This suggests that we examine the question: Does $J \mathcal{O}^{\prime}$ locally principal over $p$ insure $X$ is $a, b$-regular along $S g(X)$ at $p$ ?

First we observe that $J \mathcal{O}^{\prime}$ locally principal over $p$ implies that either $p \notin S g(X)$ or else $p \in S g(X)$ and $\operatorname{dim}_{p} S g(X)=r-1$. This is because the locus of $J$ is $\pi$ (locus of $J \mathcal{O}^{\prime}$ ). The latter either has dimension $r-1$ at some point over $p$ or is empty at every point over $p$ and $\pi$ preserves dimensions.

In what follows we sometimes require that $S g(X)$ be a manifold at $p$. By this we mean that it is a manifold with the reduced structure, not that it is a manifold with the structure induced by $J$.

A fundamental tool is the following proposition proved by John Stutz.

Proposition 3. Let $X$ be a reduced analytic space of pure dimension $r$ at $p$ with $p$ in $S g(X)$ and $S g(X)$ a manifold at $p$. Assume that $\operatorname{dim} C_{4}(X, p)=r$. Then $X$ has a Puiseux series normalization at $p$, i.e. there exists a ball $D \subset C^{r}$ and holomorphic maps $f_{j}: D \rightarrow X_{j}$ where $X_{j}$ are the irreducible components of $X$ at $p$ such that

(a) $f_{j}$ is a homeomorphism;

(b) there are coordinates $(x),(y)$ in $C^{r}$ and $C^{m}$ (the ambient space for $X$ at 
p) so that $y(p)=0$ and $f_{j}(x)=\left(x_{1}, \cdots, x_{r-1}, x_{r}^{d_{j}}, f_{r+1, j}(x), \cdots, f_{m, j}(x)\right)$, and the ball $D$ and coordinates $(x),(y)$ are independent of $j$; and

(c) $Y=C_{y_{1}, \cdots, y_{r-1}}$;

(d) if $X_{j}$ contains $S g(X)$ at $p$ then $d_{j} \leqq$ order of $f_{k, j}(x)$ in $x_{r} \forall k$.

Proof. [5, Propositions 4.2 and 4.6].

Corollary. With $X$ as in the proposition the normalization of $X$ is a manifold.

ExAmPle. The locus $X$ of $y^{2}-x^{2}(z-x)$ has singular set $C_{z}$ and normalization a manifold but $C_{4}(X, 0)$ has dimension three so the condition is not necessary.

THEOREM 4. Let $X$ be a reduced analytic space of pure dimension $r$ at $p$ with $p$ in $S g(X)$ and $S g(X)$ a manifold at $p$. Suppose that the Jacobian ideal of $X$ becomes locally principal over $p$ on the normalization of $X$. Then $X$ is $a, b$-regular along $S g(X)$ at $p$ if and only if every irreducible component of $X$ at $p$ contains $S g(X)$ at $p$. Any component of $X$ which does not contain $S g(X)$ at $p$ is a manifold at $p$.

Proof. (We understand that Stutz has also proved this theorem in a paper of his to appear in the Amer. J. Math. so we give only an indication of the proof.) Using the Jacobian matrix of the mapping $f_{j}$ of Proposition 3 it is easy to see that $S g\left(X_{j}\right) \subset Y$. If $X_{j}$ does not contain $Y, \operatorname{dim} S g\left(X_{j}\right)<$ $r-1$. By an earlier remark $S g\left(X_{j}\right)=\varnothing$ which proves the last claim of the theorem. Now the only if part follows from Hironaka's result [2, Corollary 6.2] that $a, b$-regularity implies equimultiplicity. The converse follows by careful analysis of the Jacobian matrix of the mapping $f_{j}$ of Proposition 3. Q.E.D.

EXAMPLES. It is easy to use this theorem to construct an example in which $J$ principalizes on the normalization but $X$ is not $a, b$-regular along $S g(X)$ at $p$. Zariski has given an example in which $J$ becomes principal on the normalization but $S g(X)$ is not manifold at $p$ [7, footnote 3, p. 987].

REMARKS. In [5] Stutz proved, among other things, a number of the results of this paper under additional hypotheses. He proved the equivalence of (i) and (v) of Proposition 1 assuming that $S g(X)$ is a manifold of dimension $r-1$ at $p$ and $\operatorname{dim} C_{5}(X, p)=r+1$. He proved Theorem 4 assuming $p$ simple in $S g(X), S g(X)$ a manifold of dimension $r$ at $p$, $\operatorname{dim} C_{4}(X, p)=r$, and $\operatorname{dim} C_{5}(X, p)=r+1$. We improve upon this result by applying Proposition 3 to the question of $a, b$-regularity directly rather than passing through the existence of wings as Stutz did. The assumptions used by Stutz insure that every component of $X$ at $p$ contains $S g(X)$ at $p$ but he has told us the converse is not true. Where this paper extends part 
of [5] most effectively is in the characterization of $\operatorname{dim} C_{4}(X, p)=r$ by means of the Jacobian ideal (Zariski uses this technique to get a criterion of equisingularity in [7, Theorem 5.1, p. 987].) Not only does this technique yield better results, e.g. $\operatorname{dim} C_{4}(X, p)=r$ always implies $C_{4}(X, p)=C(X, p)$ and an avoidance in Theorem 4 of the cone $C_{5}(X, p)$ which is difficult to compute, but it raises a number of interesting questions in the formal case.

Question. Suppose $R=S / \mathfrak{p}$ where $\mathfrak{p}$ is prime and $S=k\left[\left[y_{1}, \cdots, y_{n}\right]\right]$ and $R / J$ is regular and $J R^{\prime}$ is principal where $R^{\prime}$ is the integral closure of $R$. Does it follow that $R$ has a Puiseux series normalization? Is $R^{\prime}$ regular? Is the reduction of $G(R)$ a domain? These results are true in the convergent case, yet the hypothesis and conclusion are punctual but the proofs are not. We hope to turn to these questions in a subsequent paper.

We are indebted to Abhyankar who proposed the question: What are the consequences of $J$ locally principal over $p$ on the normalization of $X$ ? and to the referee who suggested we examine the relation between some of these results and the paper of Stutz.

\section{REFERENCES}

1. S. S. Abhyankar, Local analytic geometry, Pure and Appl. Math., vol. 14, Academic Press, New York, 1964. MR 31 \#173.

2. H. Hironaka, Normal cones in analytic Whitney stratifications, Inst. Hautes Études Sci. Publ. Math. No. 36 (1969), 127-138. MR 43 \#3492.

3. - Resolution of singularities of an algebraic variety over a field of characteristic zero. I, II, Ann. of Math. (2) 79 (1964), 109-326. MR 33 \#7333.

4. - - Seminar on singularities. I, Woods Hole Lecture Notes, 1964.

5. John Stutz, Analytic sets as branched coverings, Trans. Amer. Math. Soc. 166 (1972), 241-261.

6. H. Whitney, Local properties of analytic varieties, Differential and Combinatorial Topology, Princeton Univ. Press, Princeton, N.J., 1965.

7. O. Zariski, Studies in equisingularity. II. Equisingularity in codimension 1 (and characteristic zero), Amer. J. Math. 87 (1965), 972-1006. MR 33 \#125.

Department of Mathematics, George Mason University, Fairfax, Virginia 22030 